J. Appl. Cryst. (1978). 11, 496

Small-Angle X-ray and Neutron Diffraction from Corneal Stroma

(Extended Abstract only) By G. F. Elliott, J. M. Goodfellow and A. E. Woolgar, Open University Research Unit, Boars Hill, Oxford, England and P. A. Timmins, Institute Laue-Langevin, Grenoble, France

(Received 3 November 1978; accepted 8 February 1978)

The corneal stroma is a connective tissue which combines the usual properties of strength and resilience with one property, transparency, unusual in such tissues. Several models have been put forward to account for this transparency, these differ in detail but in almost all cases the regularity of arrangement of the collagen fibrils is taken to be an important parameter of the model (Maurice, 1957: Farrell \& Hart, 1969: Benedek, 1971). So far the only experimental evidence about this arrangement comes from electron microscopy, and we have therefore applied small-angle diffraction techniques because they involve less preparative procedures which might cause artifacts.

Excised stroma swells when placed in an excess of aqueous solution, and in swelling it loses its transparency. We have observed a long spacing corresponding to the centre-to-centre distance of the collagen fibrils, and have followed this spacing as a function of the hydration of the tissue with both X-ray and neutron diffraction. The spacing changes from about $40 \mathrm{~nm}$ in nearly dry tissue to as much as $130 \mathrm{~nm}$ in very hydrated tissue (hydration, i.e. ratio of water to dry weight $\sim 19$ ); the square of this interfibrillar spacing is proportional to the hydration. Changes in $\mathrm{pH}$ and ionic strength of the bathing solution affect the time course of the swelling, but our results (particularly from neutron diffraction) show that at least to a first approximation, the spacing at a given hydration does not depend on these parameters. Contrast matching experiments with neutron diffraction, where the aqueous medium has various $\mathrm{H}_{2} \mathrm{O} / \mathrm{D}_{2} \mathrm{O}$ ratios, show the interfibrillar reflection to have a minimum (effectively zero) intensity at a water content of about $45 \% \mathrm{D}_{2} \mathrm{O} / \mathrm{H}_{2} \mathrm{O}$. This is almost identical to the match point for the first-order meridional reflection from rat-tail collagen (Miller et al., 1976). As this latter reflection arises primarily from the protein-gap step function the contrast behaviour of the interfibrillar reflection shows that the fibril-interspace system behaves as if the interfibrillar space were simply an expandable aqueous medium between protein (collagen) fibrils.

We have also observed the third and fifth orders of the
$(67 \mathrm{~nm})$ collagen spacing: these do not change with tissue hydration, for example at a tissue hydration of 15 the collagen spacing observed from the third and fifth orders is 67.3 $\mathrm{nm}$, while the interfibrillar spacing is about $110 \mathrm{~nm}$. The absence of the first-order collagen spacing is surprising: we think it may be due to the occlusion of the gap between corneal collagen molecules, perhaps by the glycoproteins and glycosaminoglycans in this tissue. Calculations on the neutron scattering-length densities for collagen and for glycosaminoglycans show them to be very similar and to have similar contrast-variation behaviour in different $\mathrm{H}_{2} \mathrm{O} / \mathrm{D}_{2} \mathrm{O}$ mixtures. This would be consistent with our inability to observe the first-order meridional reflection by matching out the glycosaminoglycan contribution. (The errors in this calculation are however quite large because of the uncertainty of the specific volume of the glycosaminoglycans within the tissue.) Certainly corneal collagen fibrils are unusual in their uniform size and in their band pattern observed in the electron microscope.

In contrast the collagen fibrils of the sclera (the major nontransparent part of the eyeball), which are non-uniform in diameter, do show a first-order collagen reflection. Further details of the X-ray part of this study can be found in Goodfellow, Elliott and Woolgar (1978).

\section{References}

BenedeK, G. B. (1971). Appl. Opt. 10, 459-473.

Farrell, R. A. \& Hart, R. W. (1969). Bull. Math. Biophys. 31, 727-759.

Goodfellow, J. M., Ei.l.iott, G. F. \& Woolgar. A. E. (1978). J. Mol. Biol. 119, 237-252.

Maurice, D. M. (1957). J. Phisiol. (London), 136. 263-286. Miller, A, Brodsky Doylf, B., Hulmes, D. J. S.. Jenkin, G. T., White, J. W., HaAs, J., Ibel, K. \& Timmins, P. A. (1976). Neutron Scattering for the Analysis of Biological Structures. Brookhaven Simp. Biol. 27, III : 86-100.

J. Appl. Cryst. (1978). 11, 496-497

\title{
Small-Angle X-ray Studies of the Scaffold in Bacteriophage $\lambda$ Head Formation
}

(Extended Abstract only) By Peter KüNzler and HaNs Berger, Biozentrum der Universität Basel. Department of Structure Biology, Klingelbergstrasse 70, 4056 Basel, Switzerland

(Received 3 November 1977; accepted 8 March 1978)

The pathway of bacteriophage $\lambda$ head formation has been well characterized in vivo and in vitro (Hohn, Katsura \& Hohn, 1977). First a petit $\lambda$ particle consisting of a shell protein $\mathrm{pE}$, a protein $\mathrm{pNu} 3$ and minor proteins, but lacking DNA, is formed. The pNu3-containing structure is then converted to a structure which does not contain $\mathrm{pNu} 3$, and 